Research in Astron. Astrophys. Vol.0 (200x) No.0, 000-000

http://www.raa-journal.org http://www.iop.org/journals/raa

Research in

Astronomy and

Astrophysics

\title{
X-ray afterglow of GRB 050712: Multiple energy injections into the external shock
}

\author{
Liang-Duan $\mathrm{Liu}^{1,2}$ and A-Ming Chen ${ }^{1}$ \\ ${ }^{1}$ Institute of Astrophysics, Central China Normal University, Wuhan 430079, China \\ ${ }^{2}$ School of Astronomy and Space Science, Nanjing University, Nanjing 210093, China
}

\begin{abstract}
As indicated by the observed X-ray flares, a great amount of energy could be intermediately released from the postburst central engine of gamma-ray bursts (GRBs). As a natural consequence, the GRB external shock could be energized over and over. With such a multiple energy injection model, we explore the unique X-ray afterglow light curve of GRB 050712, which exists four apparent shallow decay plateaus. Together with three early X-ray flares, the central engine of GRB 050712 is supposed to release energy at least seven times after the burst. Furthermore we find that the energy released during four plateaus are all on the same order of magnitude, but the luminosity decreases with time significantly. These results may provide some interesting implications for the GRB central engine.
\end{abstract}

Key words: gamma rays: bursts - individual: GRB 050712

\section{INTRODUCTION}

The successful launch and operation of the Swift satellite have significantly improved our understanding of the physical origin of gamma-ray bursts (GRBs). The X-ray Telescope (XRT) onboard Swift has revealed many unexpected features in X-ray afterglows (Zhang et al. 2006), which include: (1) steep decay phase (Zhang et al. 2007; 2009), (2) shallow decay phase (Liang et al. 2007), (3) missing jet breaks in some GRBs (Liang et al. 2008; Racusin et al. 2009), and (4) X-ray flares (Falcone et al. 2007; Swenson et al. 2013). Specifically, the shallow decay segments were commonly seen in about $60 \%$ of the Swift-detected X-ray afterglows, and the flares appear in roughly 1/3 of all (Gehrels et al. 2009; Grupe 2013). It seems difficult to explain these phenomena in the standard external shock model (Mészáros et al. 1997; Sari et al. 1998; Zhang 2007; Liang et al. 2007), where a simple power-law decay with a temporal index around 1.2 is predicted in the X-ray light curves.

$\mathrm{X}$-ray flares share a lot of similarities with GRB prompt emission, which indicates that the flares might have similar physical processes with the prompt emission including the dynamics and radiation mechanisms (Fan \& Wei 2005; Zhang,B. et al. 2006; Yu \& Dai 2009). At the same time, it is widely accepted that the shallow decay afterglows could be due to an energy injection into the external shock (Dai \& Lu 1998a,b; Rees \& Mészáros 1998; Zhang \& Mészáros 2001; Fan \& Xu 2006; Nousek et al. 2006; Granot \& Kumar 2006; Sollerman et al. 2007; Geng et al. 2013). Both the X-ray plateaus and flares suggest that the GRB central engine (accreting black hole or millisecond magnetar) has not been switched off immediately after the prompt emission. For a black hole surrounded by an accretion disk, the late activities could be powered by the accretion of the fall-back materials, where the spin energy of the black hole can be released by the Blandford-Znajek mechanism (Blandford \& Znajek 1977). On the other hand, if the central engine is a millisecond magnetar, it will lose its rotational energy by some baking mechanisms (e.g., magnetic dipole radiation). 
Long GRB 050712 with a duration of $T_{90}=48 \pm 2 \mathrm{~s}$ was detected by the BAT onboard Swift at 14:00:28 UT on 2005 July 12, and the XRT refined coordinates RA(J2000) = $05^{\mathrm{h}} 10^{\mathrm{m}} 48^{\mathrm{s}}$, Dec $(\mathrm{J} 2000)=64^{\circ} 55^{\prime} 48.2^{\prime \prime}$ with a $6^{\prime \prime}$ radius error circle. Its peak flux measured in the $15-150 \mathrm{keV}$ band was $(1.10 \pm 0.07) \times 10^{-6} \mathrm{erg} \mathrm{cm}^{-2} \mathrm{~s}^{-1}$. The XRT observation in the $0.3-10 \mathrm{keV}$ band started at about $160 \mathrm{~s}$ after the trigger. The observed X-ray light curve exhibits a unique temporal behavior. Firstly, three flares happened at 174, 455 and 861s (Swenson \& Roming 2013) after the BAT trigger respectively. Such early flares are usual in Swift GRBs (Falcone et al 2007; Zhang,B. et al 2006). However, after these flares, the afterglow emission declines tierdly (i.e., step by step; see Figure 1), which is completely different from the normal single-power-law decay or a single plateau followed by a power-law decay. Furthermore, the rise and decline of these steps are very slow, so they can not be late flares. Obviously, such a tierd light curve can not be explained by the traditional energy injection model where the energy is continuously injected. Alternatively, in this paper we suggest that the afterglow emission of GRB 050712 could indicate several intermediate, multiple energy injections.

\section{MODEL}

In the standard model of GRBs (Zhang 2007; Gehrels et al. 2009), the GRB central engine drives an energetic fireball or Poynting-flux-dominated ejecta, which could be accelerated to a high relativistic speed. Then the internal dissipations in the ejecta (e.g. internal shocks and magnetic reconnections) lead to the prompt gamma-ray emission. Subsequently, the interaction between the GRB ejecta and the ambient medium gives rise to a relativistic external shock, which could produce long-lasting multiwavelength afterglow emission. Therefore, the temporal behavior of the afterglow emission should be basically determined by the dynamical evolution of the external shock. A generic dynamical model which covers both the ultra-relativistic and non-relativistic shock dynamics was delineated by Huang et al.(1999;2000b). The fundamental dynamical equation can be written as

$$
\frac{d \Gamma}{d m_{\mathrm{sw}}}=-\frac{\Gamma^{2}-1}{M_{\mathrm{ej}}+2 \Gamma m_{\mathrm{sw}}},
$$

where $\Gamma$ is the bulk Lorentz factor of the external shock, $m_{\mathrm{sw}}$ is the mass of the swept-up medium, and $M_{\mathrm{ej}}$ is the mass of the GRB ejecta.

The post-burst energy injection into the external shock could significantly slow down the deceleration rate of the shock or even lead to a short-term acceleration (Dai \& Liu 2012). The dynamical equation can be derived from the energy conservation law. The total energy of the GRB ejecta and the shocked medium can be written as $E_{k}=\Gamma M_{\mathrm{ej}} c^{2}+\left(\Gamma^{2}-1\right) m_{\mathrm{sw}} c^{2}$, where the shock jump condition is used to calculated the internal energy. Then we can get $d E_{k}=\left(\Gamma^{2}-1\right) c^{2} d m_{\mathrm{sw}}+\left(M_{\mathrm{ej}}+2 \Gamma m_{\mathrm{sw}}\right) c^{2} d \Gamma=$ $L(t) d t$, where $L$ is the luminosity of the post-burst energy flow released from the central engine and $t=t_{\mathrm{obs}} /(1+z)$ with $t_{\mathrm{obs}}$ being the time measured in the observer's frame and $z$ redshift (Fan $\& \mathrm{Xu}$ 2006). Therefore, we can revise the dynamical equation as follows

$$
\frac{d \Gamma}{d m_{\mathrm{sw}}}=-\frac{1}{M_{\mathrm{ej}}+2 \Gamma m_{\mathrm{sw}}}\left[\Gamma^{2}-1-\frac{L(t)}{c^{2}} \frac{d t}{d m_{\mathrm{sw}}}\right] .
$$

If $L=0$ (no energy injection), the above equation returns to Eq. (1). For a general consideration, Zhang \& Mészáros (2001) proposed a simple power-law form for the injected luminosity as $L(t) \propto t^{-q}$. Specifically, $q=5 / 3$ is expected if the central object is a black hole surrounded by a fallback accretion disk. However, for $q>1$, the energy injection is not important. On the other hand, for a millisecond magnetar, $q=0$ or -2 can be found for a traditional magnetic dipole radiation before/after the spin down timescale (Dai \& Lu 1998a). Moreover, for a fallback-accreting magnetar, its spin down history could become much more complicated (Dai \& Liu 2012) and then the value of $q$ might evolve with time.

In most energy injection models, the energy release is usually assumed to be continuous. However, as indicated by the X-ray flares, the energy release from the post-burst object could be intermediate. It seems that the central engine can be turned on after a period of energy accumulation. Therefore, it is 


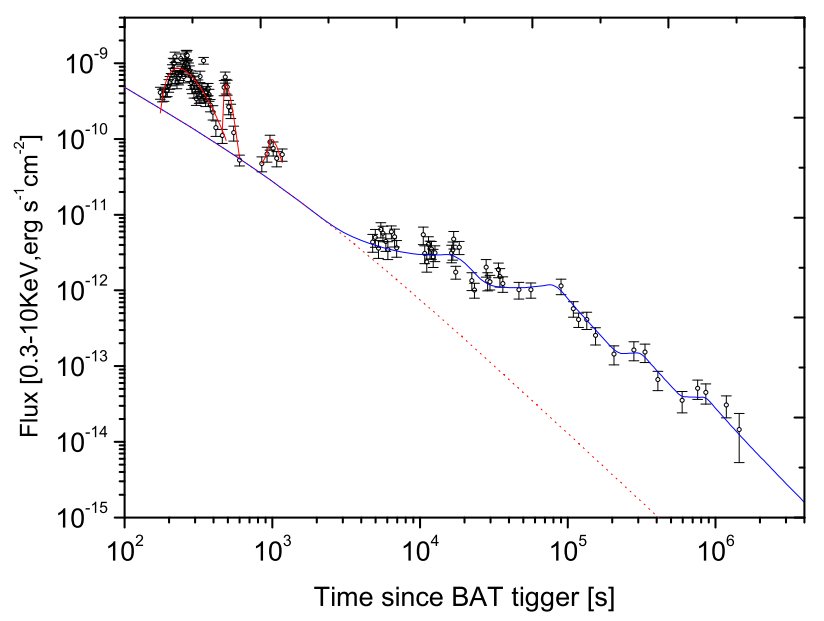

Fig. 1: Fitting to the X-ray afterglow of GRB 050712 with multiple energy injections (solid blue line), where the case without energy injection is also presented for a comparison (dotted line). The three early flares are fitted by the empirical "Norris function" function (solid red line). The observational data are taken from http : //www.swift.ac.uk/xrt_curves/00145581/.

reasonable to consider that the energy injection into the external shock might be intermediate. In this case, we have

$$
L_{i}(t)=Q_{i} t^{q_{i}}, \text { for } t_{\text {strat }, i}<t<t_{\mathrm{end}, i}
$$

where the subscript " $i$ " represents the $i$ th energy injection, $Q_{i}$ and $q_{i}$ are constants in each injection, and the energy injection starts and ends at $t=t_{\text {start }, i}$ and $t_{\mathrm{end}, i}$ respectively. For an example, Geng et al. (2013) used a two-step energy injection model to interpret the rebrightening of the multi-band afterglow of GRB 081029.

In order to solve equation (2), the evolutions of the swept-up mass $m_{\mathrm{Sw}}$ and the radius $r$ of the shock should be described together as (Huang et al. 1999, 2000b)

$$
\begin{gathered}
\frac{d m_{\mathrm{sw}}}{d r}=2 \pi r^{2}\left(1-\cos \theta_{j}\right) n m_{\mathrm{p}}, \\
\frac{d r}{d t}=\frac{\beta c}{1-\beta},
\end{gathered}
$$

where $n$ is the ambient number density, $\theta_{j}$ the half opening angle of the jet, $m_{p}$ the mass of proton, and $\beta=\sqrt{1-\Gamma^{-2}}$ the velocity in the unity of the speed of light. The distribution of the ambient medium plays an important role in determining the profile of the afterglow light curve. On contrary to the usually assumed homogenous interstellar medium (ISM), a wind-like circumstance with $n \propto r^{-2}$ is also proposed by Dai \& Lu (1998c) and Chevalier \& Li (2000) by considering that long GRBs probably originate from the collapse of massive stars (Woosley 1993; MacFadyen \& Woosley 1999).

As the propagation of the external shock, charge particles are accelerated and the stochastic magnetic field is amplified. The internal energy of the shocked medium is shared by magnetic fields and electrons with fractions $\epsilon_{B}$ and $\epsilon_{e} \sim \sqrt{\epsilon_{B}}$ (Medvedev 2006), respectively. The accelerated electrons are further assumed to distribute as $n_{\gamma^{\prime}}^{\prime} \propto \gamma^{\prime-p}$ (for $\gamma_{m}^{\prime} \leq \gamma^{\prime} \leq \gamma_{M}^{\prime}$ ) with the minimum and maximum electron Lorentz factors $\gamma_{m}^{\prime}=\epsilon_{e} \frac{m_{p}(p-2)}{m_{e}(p-1)}(\Gamma-1)$ and $\gamma_{M}^{\prime} \sim q_{e} B^{\prime} r /\left(m_{e} c^{2}\right)$, where the prime represents that the quantities are measured in the comoving frame. The magnetic filed strength $B^{\prime}$ can be 


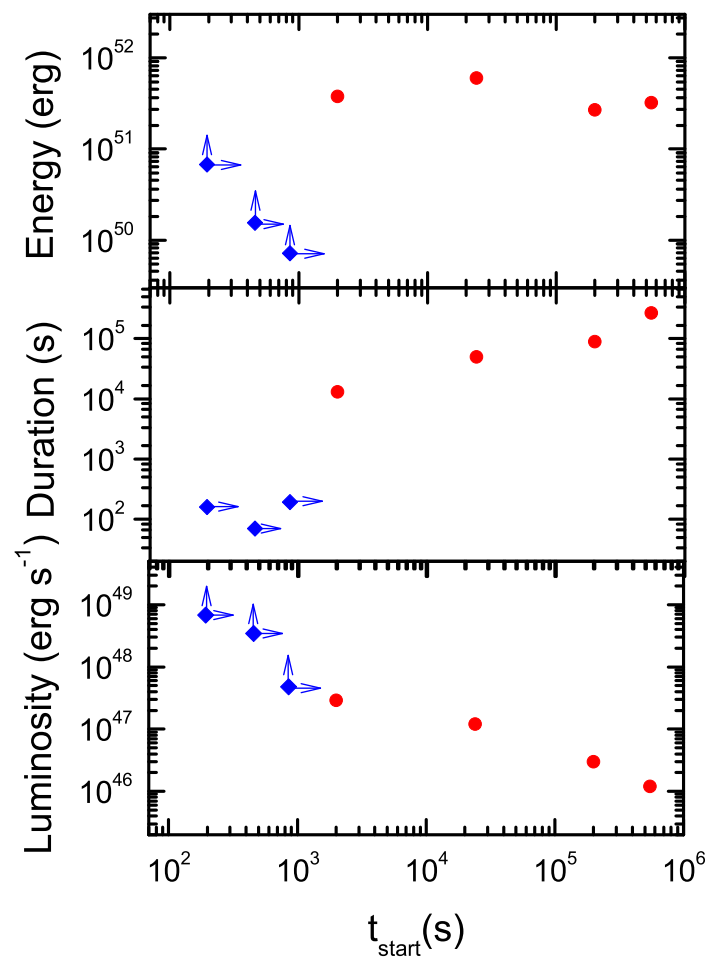

Fig. 2: The energy (top), duration (middle), and luminosity (bottom) of the energy releases by the central engine during three flares (diamonds) and the four plateaus (circles).

calculated with the parameter $\epsilon_{B}$. By considering the radiative cooling of the electrons and introducing a cooling Lorentz factor $\gamma^{\prime}{ }_{c}=6 \pi m_{e} c /\left(\sigma_{T} B^{\prime 2} \Gamma t\right)$, where $\sigma_{T}$ is Thomson cross section, a quasi-steady electron distribution can be written as (Huang et al. 2000b)

$$
n_{\gamma^{\prime}}^{\prime}=\frac{n_{e}^{\prime}}{\gamma_{l}^{\prime} l} \begin{cases}\left(\frac{\gamma^{\prime}}{\gamma^{\prime}{ }_{l}}\right)^{-x}, & \gamma_{l}^{\prime} \leq \gamma^{\prime} \leq \gamma_{h}^{\prime}, \\ \left(\frac{\gamma_{h}^{\prime}}{\gamma^{\prime}{ }_{l}}\right)^{-x}\left(\frac{\gamma^{\prime}}{\gamma_{h}{ }_{h}}\right)^{-p-1}, \gamma_{h}^{\prime}<\gamma^{\prime} \leq \gamma_{M}^{\prime} .\end{cases}
$$

For $\gamma_{c}^{\prime}>\gamma_{m}^{\prime}$ (slow cooling), we have $x=p, \gamma_{l}^{\prime}=\gamma_{m}^{\prime}$, and $\gamma_{h}^{\prime}=\gamma_{c}^{\prime}$, whereas $x=2, \gamma_{l}^{\prime}=\gamma_{c}^{\prime}$ and $\gamma_{h}^{\prime}=\gamma_{m}^{\prime}$ for $\gamma_{m}^{\prime}>\gamma_{c}^{\prime}$ (fast cooling). The comoving synchrotron emission coefficient at frequency $\nu^{\prime}$ can be given by (Rybicki \& Lightman 1979)

$$
j_{\nu^{\prime}}^{\prime}=\frac{1}{4 \pi} \frac{\sqrt{3} q_{e}^{3} B^{\prime}}{m_{e} c^{2}} \int_{\gamma_{l}^{\prime}}^{\gamma_{M}^{\prime}} n_{\gamma^{\prime}}^{\prime}\left[\frac{\nu^{\prime}}{\nu^{\prime}{ }_{0}} \int_{\frac{\nu^{\prime}}{\nu^{\prime} 0}}^{\infty} K_{5 / 3}(t) d t\right] d \gamma^{\prime},
$$

where $\nu_{0}^{\prime}=3 q_{e} B^{\prime} \gamma^{\prime 2} /\left(4 \pi m_{e} c\right)$ and $K_{5 / 3}(t)$ is the Bessel function. Then the synchrotron flux density received by the observers can be calculated as

$$
F_{\nu}(t)=\frac{(1+z)}{d_{L}^{2}} \int_{0}^{\theta_{j}} \frac{j^{\prime}{ }_{\nu^{\prime}} V^{\prime}}{\Gamma^{3}(1-\beta \cos \theta)^{3}} \frac{\sin \theta}{2} \cos \theta d \theta,
$$


Table 1: Parameters for the energy injections

\begin{tabular}{c|ccc}
\hline \hline the $i$ th injection & $t_{\text {start }}\left(10^{4} \mathrm{~s}\right)$ & $t_{\text {end }}\left(10^{4} \mathrm{~s}\right)$ & $Q\left(10^{46} \mathrm{erg} \mathrm{s}^{-1}\right)$ \\
\hline 1 & 0.3 & 1.4 & 15.0 \\
\hline 2 & 2.0 & 7.3 & 5.0 \\
\hline 3 & 19.0 & 28.0 & 1.1 \\
\hline 4 & 55.0 & 75.0 & 1.2 \\
\hline
\end{tabular}

where $\nu=\nu^{\prime} /[\Gamma(1-\beta \cos \theta)]$ is the observational frequency, $d_{L}$ is the luminosity distance of the GRB, and $V^{\prime}$ is the comoving volume of emission region. Additionally, the equal-arrival-time effect has also been taken into account in our calculation.

\section{NUMERICAL RESULTS}

We use the model described above to reproduce the light curve of the X-ray afterglow of GRB 050712. A comparison between the numerical result with the observational data is presented in Figure 1. As shown, the unusual tierd X-ray light curve requires four times of independent energy injections into the external shock. The reduced chi-square $\chi^{2} / \nu$ of the fitting is 1.253 . The model parameters are taken as follows. (1) GRB ejecta parameters: the initial isotropic-equivalent kinetic energy $E_{0}=2.5 \times 10^{51} \mathrm{erg}$, the initial Lorentz factor $\Gamma_{0}=125$, and the half opening angle $\theta_{j}=0.1$; (2) Microphysical parameters: $\epsilon_{B}=0.04, \epsilon_{e}=0.2$, and $p=2.3$; (3) Circumstance Parameter: $A=3 \times 10^{35}$, where a wind-like circumstance is taken because a too high value of $p$ is required in the ISM case which is inconsistent with the observed spectral index; (4) Energy injection parameters: as listed in Table 1, where $q=0$ is adopted in all of the four injections; (5) Redshift: $z=1$, where the average value of the Swift GRBs is taken because there is no redshift measurement for GRB 050712. It should be noted that the goodness of the fitting here is only judged by the eye, but not by a precise statistical criterion, as usually did in GRB afterglow modelings, so that the error bars of the parameters can not be given. This because too many parameters are involved and a more precise fitting is in fact not more informative. In any case the main features of the light curve has been captured by our fitting. Additionally, the three early flares are fitted by the "Norris function" as $f(t)=C \exp \left(-\tau_{1} /\left(t-t_{0}\right)-\left(t-t_{0}\right) / \tau_{2}\right)$, where $C$ is the intensity, $t_{0}$ is the beginning time, $\tau_{1}$ and $\tau_{2}$ are the rise and decay timescales, respectively (Norris et al. 2005). The obtained luminosity and integrated energy of the flares can be regarded as a lower limit because the emission efficiencies of the flares are unknown.

The three early flares and the following four energy injections indicate that the central engine of GRB 050712 releases energy at least seven times after the burst. The energy, duration, and luminosity of these energy releases are given in Figure 2, where the energy and luminosity during the flares are represented. Swenson \& Roming (2013) gave the flares of GRB 050712 start time lower limit. As it is seen, the injection of energy at different times is comparable to each other on the order of magnitude of $3 \times 10^{51} \mathrm{erg}$. The duration $\left(t_{\text {end,i } i}-t_{\text {start }, i}\right)$ of the energy released increases with time and thus the luminosity $\left(Q_{i}\right)$ declines. These features could have important implications for the properties of the central engine.

\section{SUMMARY AND DISCUSSION}

GRB 050712 is one of the most interesting Swift GRBs, whose X-ray afterglow has four apparent shallow decay plateaus. In this paper, we numerically calculated the dynamical evolution and the radiation of the GRB external shock with multiple energy injections. The model can explain the X-ray afterglow light curve of GRB 050712 well. This indicates that the central engine of GRB 050712 intermediately releases energy at least seven times after the burst, and the first three ones produce X-ray flares directly via internal dissipation and the latter four ones result in four plateaus by injecting energy into the external shock. The energy released during these four plateaus are similar, but the luminosity decreases significantly with an increasing duration. Since the close connection between the energy injections and 
the late activities of the central engine, our result demonstrates that the GRBs with multiple energy injections can be used to explore the properties of the GRB central engine. At least, our result indicates that the GRB central engine can last much longer than thought, which is related to the $t_{\text {burst }}$ topic discussed in Zhang et al (2013). In addition, such a multiple energy injection model may also have further implications for the central-object-powered supernovae (Kasen \& Bildsten 2010) and mergernovae (Yu et al. 2013).

For a single or a few plateaus, the plateau emission can in principle be ascribed to a reverse shock propagating into the slow tail of GRB ejecta (e.g., Uhm et al. 2012; Gao et al. 2013). The tierd X-ray afterglow of GRB 050712 presented here unambiguously break the degeneracy between the reverse shock model and the energy injection model. Nevertheless, in this paper, we simply assumed that the released energy is completely injected into the GRB external shock. The energy released from the central engine is probably in the form of Poynting flux initially, which could be accelerated and thermalized before it catches up with the GRB external shock. Therefore, the internal dissipation of the energy flow could directly produces some afterglow emission such as the so-called internal-origin afterglow (Yu et al. 2010; Zhang 2013). The internal dissipation may arise from turbulent magnetic reconnection (Zhang \& Yan 2011) or from the termination shock of the injected flow arising from the collision between the injected flow and the GRB ejecta (Dai 2004; Yu \& Dai 2007; Yu et al. 2007; Mao et al. 2010; Dai \& Liu 2012).

Acknowledgements This work made use of data supplied by the UK Swift Science Data Centre at the University of Leicester. The authors thank the instruction of Yun-Wei Yu who motivated this work. This work is supported by the National Natural Science Foundation of China (Grant No. 11103004), the Funding for the Authors of National Excellent Doctoral Dissertations of China (Grant No. 201225), and the Program for New Century Excellent Talents in University (Grant No. NCET-13-0822).

\section{References}

Blandford, R. D., \& Znajek, R. L. 1977, MNRAS, 179, 433

Chevalier,R. A., \& Li, Z. Y. 2000, ApJ, 536, 195

Dai, Z. G., 2004, ApJ, 606, 1000

Dai, Z. G., \& Lu, T. 1998a, A\&A, 333, 87

Dai, Z. G., \& Lu, T. 1998b, Physical Review Letters, 81, 4301

Dai, Z. G., \& Lu, T. 1998c, MNRAS, 298, 87

Dai, Z. G., \& Liu, R. Y. 2012, ApJ, 759, 58

Faclone, A. D., Morris, D., Racusin, J., et al. 2007, ApJ, 671, 1921

Fan, Y.-Z., \& Wei, D. M., 2005, MNRAS, 364, 42

Fan, Y.-Z., \& Xu, D. 2006, MNRAS, 372, L19

Gao, H., Lei, W.-H., Zou, Y.-C., Wu, X.-F., \& Zhang, B. 2013, New Astron. Rev., 57, 141

Gehrels, N., Ramirez-Ruiz, E., \&Fox, D. B. 2009, ARA\&A, 47, 567

Geng, J. J., Wu, X. F., Huang, Y. F., \& Yu, Y. B. 2013, ApJ, 779, 28

Granot, J., \& Kumar, P. 2006, MNRAS, 366, L13

Grupe, D., Nousek, J. A., Veres, P., Zhang, B.-B., \& Gehrels, N. 2013, ApJS, 209, 20

Grupe, D., Barthelmly, s., et al. 2005a, GCN 3573

Grupe, D., Burrows, D., Morris, D,. et al. 2005b, GCN 3579

Huang, Y. F., Dai, Z. G., \& Lu, T. 1999, MNRAS, 309, 513

Huang, Y. F., Dai, Z. G., \& Lu, T. 2000a, MNRAS, 316, 943

Huang, Y. F., Gou, L. J., Dai, Z. G., \& Lu, T. 2000b, ApJ, 543, 90

Kasen, D., \& Bildsten, L. 2010, ApJ, 717, 245

Kong, S. W., \& Huang, Y. F. 2010, Science China-Physics, Mechanics \& Astronomy, 53, 94

Liang, E.-W., Racusin, J. L., Zhang, B., Zhang, B.-B., \& Burrows, D. N. 2008, ApJ, 675, 528

Liang, E.-W., Zhang, B.-B., \& Zhang, B. 2007, ApJ, 670, 565

MacFadyen, A. I., \& Woosley, S. E. 1999, ApJ, 524, 262 
Mao, Z., Yu, Y. W., Dai, Z. G., et al. 2010, A\&A, 518, 27

Medvedev, M. V. 2006, ApJ, 651, L9

Meszaros, P., \& Rees, M. J. 1997, ApJ, 476, 232

Norris, J. P., Bonnell, J. T., Kazanas, D., et al. 2005, ApJ, 627, 324

Nousek, J. A., Kouveliotou, C., Grupe, D., et al. 2006, ApJ, 642, 389

Racusin, J. L., Liang, E. W., Burrows, D. N., et al. 2009, ApJ, 698, 43

Rees, M. J., \& Meszaros, P. 1998, ApJ, 496, L1

Rybicki, G. B., \& Lightman, A. P. 1979, New York, Wiley-Interscience, 1979. 393 p.,

Sari, R., Piran, T., \& Narayan, R. 1998, ApJ, 497, L17

Sollerman, J., Fynbo, J. P. U., Gorosabel, J., et al. 2007, A\&A, 466, 839

Swenson, C. A., \& Roming, P. W. A. 2013, arXiv:1310.2628

Uhm, Z. L., Zhang, B., Hascoët, R., et al. 2012, ApJ, 761, 147

Woosley, S. E. 1993, ApJ, 405, 273

Yu, Y. B. \& Huang, Y. F. 2013, Research in Astron. Astrophys. (RAA), 13, 662

Yu, Y. W., Cheng, K. S. \& Cao, X. F. 2010, ApJ, 715, 477

Yu, Y. W. \& Dai, Z. G. 2007, A\&A, 470, 119

Yu, Y. W. \& Dai, Z. G. 2009, ApJ, 692, 133

Yu, Y. W., Liu, X. W., Dai, Z. G. 2007, ApJ, 671, 637

Yu, Y.-W., Zhang, B., \& Gao, H. 2013, ApJ, 776, L40

Zhang, B. 2007, ChJAA (Chin. J. Astron. Astrophys.), 7, 1

Zhang, B. 2013, ApJL, 763, L22

Zhang, B., Fan, Y. Z., Dyks,J., et al. 2006, ApJ, 642, 354

Zhang, B., Liang, E., Page, K. L., et al. 2007, ApJ, 655, 989

Zhang, B., \& Mészáros, P. 2001, ApJ, 552, L35

Zhang, B., Yan, H. 2011, ApJ, 726, 90

Zhang, B.-B., Liang, E.-W., \& Zhang, B. 2007, ApJ, 666, 1002

Zhang, B.-B., Zhang, B., Liang, E.-W., \& Wang, X.-Y. 2009, ApJ, 690, L10

Zhang, B.-B., Zhang, B., Murase, K., Connaughton, V., \& Briggs, M. S. 2013, arXiv:1310.2540 